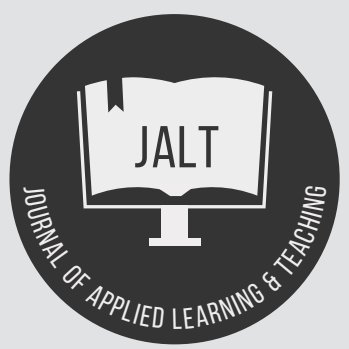

Vol.3 No.2 (2020)

\title{
Journal of Applied Learning \& Teaching
}

ISSN : 2591-801X

Content Available at : http://journals.sfu.ca/jalt/index.php/jalt/index

Virtual Reality as a tool for learning: The past, present and the prospect

\begin{tabular}{l} 
Yewande M. A \\
Oluwatoyin C. \\
Oluwafemi A. \\
\hline Keywords \\
\hline Learning; \\
training; \\
virtual laboratory; \\
virtual reality.
\end{tabular}

\section{Article Info}

Received 17 July 2020

Received in revised form 26 August 2020

Accepted 27 August 2020

Available online 27 August 2020

DOI: https://doi.org/10.37074/jalt.2020.3.2.10
Department of Computer Science, The Federal University of Technology, Akure-Nigeria

Department of Information Systems, The Federal University of Technology, Akure-Nigeria

Department of Computer Science, The Federal University of Technology, Akure-Nigeria

\begin{abstract}
The use of computing technologies in human learning is rapidly growing and advancing in various fields of learning and training. Virtual reality (VR) is one of the growing computer techniques used in schools and training institutes to help improve students' learning experience, create an interactive environment and build students' confidence while working in a physical environment. The benefits of using virtual reality are yet to be fully explored in all fields of endeavour. Virtual reality has been applied in the field of medicine for rehabilitation of patients and in training of medical students. In addition, it has been used in operations management, manufacturing processes and design as well as in the aviation industry for the dissemination of safety information, and maintenance. VR holds great and promising prospects in education, tourism, entertainment, and architecture. Hence, this paper presents a review of the trends of applications of virtual reality technologies, its potentials and prospects for learning in various fields.
\end{abstract}




\section{Introduction}

Virtual Reality (VR) refers to an immersive, interactive, multisensory, viewer-centered, three-dimensional computer generated environment that requires the combination of technologies to build such environments (Mazuryk \& Origin of VR Technology.

According to Ellis (1994), the development of VR systems can be traced to the developments of vehicle simulation. The head-mounted periscope display was invented in 1916. It was the first of its kind to use a VR system. The headmounted periscope displays developed by Continental Aviation and Engineering (CAE) are fibre-optic helmetmounted displays which were designed to replace the bulky, dome-projection flight simulators. The work in vehicle simulation goes back to the work of Edwin Link in the late 1920's (Ellis, 1994). In 1929, a simple and mechanical device used as an instrument in flight training was developed for the first time which was termed a flight simulator (Baarspul, 1990). Furthermore, the Teleoperation technology was developed next in the 1940's with its system components developed during the early 1960's (Ellis, 1994). In the early 1950's, cinematographer Morton Heilig developed the multi-sensory simulator (Sensorama) with wind and scent production, vibratory sensation and 3D display (Drummond et al., 2014; Martirosov \& Kopecek, 2017). Sensorama was an early head-mounted display which uses 3D visual, audio, haptic, olfactory stimuli to view 3D photographic slides, and was used in the tracking of head orientation and in creating an immersive experience (Mazuryk \& Gervautz, 1996; Boas, 2013). Heilig also invented the Telesphere Mask that was patented in 1960. The Telesphere Mask was the first head mounted device (HMD). The headset provided stereoscopic 3D and wide vision with stereo sound (Mazuryk \& Gervautz, 1996). Two Philco Corporation engineers, Comeau and Bryan developed the first precursor to the HMD known as Headsight in 1961. The Headsight is a helmet that has a video screen for each eye, a magnetic motion tracking system, cathode ray tube display and a tracking system to identify head position (Boas, 2013).

Further work by Ivan Sutherland pioneered the personalised graphics simulation which led to the first synthetic computergenerated display used in virtual environments. In 1963, Ivan Sutherland developed a Sketchpad which was the first interactive computer graphics item. The sketchpad uses a man-machine graphical communications system and a light pen to perform selection and drawing interactions (Mazuryk \& Gervautz, 1996). In 1966, Thomas A. Furness III introduced a visual flight simulator for the Air Force (Kumar, 2014). Ivan Sutherland in 1968 advanced our knowledge in sketchpads with the invention of the "Ultimate Display". The Ultimate Display was the first computer-aided HMD with internal sensors that tracked the user's head movement (Mazuryk \& Gervautz, 1996; Dixon, 2006; Kumar, 2014). Furthermore, Ivan Sutherland, developed a VR system in the form of a hardware "The Sword of Damocles" in 1968. This VR system hardware was hung on the ceiling because it was big in size but had an appropriate head tracking system (Mazuryk \& Gervautz, 1996, Boas, 2013).
Scholars from the University of North Carolina (UNC) in 1971 were able to develop the first prototype of a forcefeedback system called GROPE (Mazuryk \& Gervautz, 1996). In 1972, General Electric Corporation built a computerised flight simulator which featured a 180-degree field of vision by using three screens surrounding the cockpit (Mazuryk \& Gervautz, 1996). Myron Krueger in 1975 invented an Artificial Reality system termed "VIDEOPLACE" which is a conceptual environment that had never been in existence. In this system, the silhouettes of the user are captured by the cameras and were projected on a large screen (Mazuryk \& Gervautz, 1996). Aspen Movie Map was created in 1977 at the Massachusetts Institute of Technology. Aspen Movie Map was a crude virtual simulation of Aspen in which users could maneuvre the streets of Colorado. Also, in 1977 Tom DeFanti and Daniel J. Sandin invented Wired Gloves which worked with fibre-optics and the first one created were the Sayre glove (Boas, 2013).

McDonnell-Douglas Corporation integrated VR into its HMD (the VITAL helmet) for use in the military. A head tracker in the HMD followed the pilot's eye movements to match the computer-generated images. An advanced flight simulator was developed at the US Air Force's Aerospace Medical Research Laboratories (AFAMRL) by Thomas Furness. He developed the Visually Coupled Airborne Systems Simulator (VCASS) in 1982, visually coupled systems facilities for in flight, simulated control of threat and weapon systems (Mazuryk \& Gervautz, 1996; Welch, 2009). Similarly, in 1984, the Virtual Visual Environment Display was designed at the NASA Ames, USA. This was a stereoscopic monochrome HMD - an off-the-shelf technology at the time (Mazuryk \& Gervautz, 1996). The Virtual Programming Lab (VPL) company founded by Jaron Lanier in 1983 fabricated the first commercially available VR devices popularly known as DataGlove in 1984 and the Eyephone HMD in 1988 (Mazuryk \& Gervautz, 1996, Dixon, 2006). The DataGlove heavily influenced the manufacturing of other devices such as the Power Glove by Mattel for the Nintendo Entertainment System in the 1980's (Boas, 2013). The VPL Company together with Thomas Zimmerman in 1986 developed wired gloves which enabled virtual objects to be grasped and moved. In 1986, Frederick Brooks developed Grope-III project which allowed a sense of touch within the VR using motorised hand grips and magnets that controlled remote robotic arms (Dixon, 2006). In 1989, a Binocular OmniOrientation Monitor (BOOM) was commercialised by Fake Space Labs. BOOM is a small box containing two cathoderay tube (CRT) monitors that can be viewed through the eye holes (Mazuryk \& Gervautz, 1996).

In the second half of the 1980's at the University of North Carolina, an architectural walkthrough application was developed, called the UNC Walkthrough project. Several VR devices were constructed to improve the quality of this system which includes: head mounted devices (HMDs), optical trackers and the Pixel-Plane graphics engine (Mazuryk \& Gervautz, 1996). In the early 1990's, Virtual Wind Tunnel was developed at NASA, Ames, USA. This application allows the observation and investigation of flow-fields with the help of BOOM and DataGlove (Mazuryk \& Gervautz, 1996). In 1991 Antonio Medina, an MIT graduate and NASA scientist, designed Computer Simulated Teleoperation - a 
VR system that allows the piloting of a Mars robot rover from Earth taking into account the time delay (Kumar, 2014). In 1992, Daniel Sandin and Thomas DiFanti developed CAVE (cave automatic virtual environment) which is a scientific visualisation system which uses immersive projection onto three walls and the floor of a room, although stereoscopic glasses are worn (Dixon, 2006). However, the users need to put on stereoscopic glasses, use a wand mouse to manipulate the environment and a head tracker to detect the user's changing spatial position in order to display a realistically changing perspective (Mazuryk \& Gervautz, 1996; Dixon, 2006).

The ImmersaDesk was developed in 1996. In July 1995, Nintendo's R\&D1 group, spearheaded by famed Gumpei Yokoi, launched the Nintendo Virtual Boy console which played 3D monochrome video games. It was the first and only dedicated stereoscopic portable console to display 3D graphics (Zachara \& Zagal, 2009). In 1997, Georgia Tech and Emory University collaborated to use VR for the treatment of PTSD in war veterans (Rizzo et al., 2008) In 2007, Google introduced Street-view which an alternative source of data while it enhances online Maps service with street-level 360-degree pan images, video footage captured by cars fitted with custom camera equipment (Rundle et al., 2011). Similarly, Google introduced a stereoscopic 3D mode for street-view by Palmer Lucky in 2010 who presented the first prototype for Oculus Rift (Mykhailovska et al., 2019). In 2014, Sony announced Project Morpheus, a VR system which leverages the PlayStation4's (PS4) outstanding graphics computing power (Markwalter, 2014). HTC released its HTC VIVE SteamVR headset in 2016. The HTC Vive is designed to turn a room into a 3D space which allows users to move freely in a space (Egger et al., 2017) while in 2018, Facebook F8 announced a new headset prototype called Half Dome with a varifocal function (Mun et al., 2018).

Several advances have been made in the area of VR as reported by Mazuryk and Gervautz, (1996); Potkonjak et al. (2016). VR is now being used in a variety of ways, from providing immersive gaming experiences, to helping treat psychological disorders, to teaching new skills and even taking terminally ill people on virtual journeys. VR has many applications and with the rise in smartphone technology VR will be even more accessible (Gervautz, 1996). VR is also a technology, a communication interface and an environment that provides interactive experience (Riva, 2003). The field of VR has grown enormously and the practical applications of the VR technology has been reported in many fields (Holden, 2005). This accounts for the rise in the use of VR and its technology in various fields. Nowadays, research and commercial VR systems are used for simulation and training, industrial design, phobia therapy and other health-related applications, surgical planning and assistance, artistic applications, and in games (Welch, 2009).

The development of a VR system involves the collection of technological hardware, including computers, headmounted displays, headphones, and motion-sensing gloves (Steuer, 1992). VR provides a unique medium suited to the achievement of several requirements for effective rehabilitation intervention in medical treatment (Sveistrup, 2004). Virtual laboratories address the lack of laboratory infrastructure in most high schools and community colleges, especially in areas with low socio-economic status (Desai et al., 2017). The application of VR as an aided learning technology ranged from aviation training, military, industrial machine operations and in medicine where surgeons can be trained in surgical techniques through the VR systems (Holden, 2005). Brown and Standen (2006) also reported the potential of VR as an educational tool for those with intellectual disabilities. It is thought that students are better able to master, keep in mind, and generalise new knowledge when actively involved in the creation of knowledge. This idea is termed constructivism according to the philosophy of pedagogy (Youngblut, 1998). Science is obviously connected to technology cognitively and practically (Babateen, 2011). In order to make simple, reduce risks, minimise time of completion and cost of some experiments in the educational sector and other sectors, professionals have studied the integration of both information and communication technology for a better learning experience (Babateen, 2011).

Hence, VR is considered to be a new model of computerbased learning that provides the individual learner with a broader range of scientific vision (Chow \& Andrews, 2007; Babateen, 2011). Virtual environment displays interactive head-referenced computer displays that give users the illusion of displacement to another location. Virtual environments potentially provide a new communication medium for human-machine interaction (Ellis, 1994). The VR environments allow users to interact with objects and environments that ordinarily will not be possible. Virtual environments are considered to be a perfect environment for testing phenomena that may be too costly or too dangerous in physical reality (Shudayfat $\&$ Moldoveanu, 2012).

In the physical environment, students can learn and configure personal scientific knowledge in the laboratories. Laboratory activities which are integral components of science lessons enable students to build up their own experience using real materials (Tatli \& Ayas, 2010). An alternative learning environment to physical laboratory learning is the virtual laboratory system, which contributes to the occurrence of meaningful learning (Bortnik et al., 2017). The virtual laboratories open up a wide range of experiments to audiences that would otherwise not be made possible (Schmid, 2017). Virtual laboratories are used in varied science programmes, especially to achieve a handson practical experience (Lambropoulos, 2007). Multimedia virtual laboratories are used to aid understanding of resource material that could provide solutions as well as overcome the restrictions associated with instrumentation devices in a real lab (Zurweni et al., 2017). In this paper, we discuss the benefits of VR as an aided learning tool, for training, and an alternative medium for human experience, its applications, the technological advancement and potential future applications.

\section{Advantages and Disadvantages of VR Technology in Learning Environments}

With the rapid growth of VR and its application in various fields, it is important to specify the advantages of VR 
over the physical facilities while pointing out its potential disadvantages.

\section{Advantages}

VR systems provide a cost-efficient way of passing knowledge across in learning environments such as high schools, universities and science laboratories and in a variety of disciplines (Potkonjak et al., 2016), thus creating avenues for cost-savings. VR affords the opportunity for flexibility in learning environments. The conduct of laboratory experiments often requires hazardous reagents and apparatuses that might not be easily accessible and affordable, hence a virtual environment can easily be created to overcome these challenges (Potkonjak et al., 2016). Multimodal Collaborative Virtual laboratories (MMCVL) are virtual chemistry labs designed to address the problem of lack of resources and safe use of expensive laboratory equipment (Desai et al., 2017).

The amazing benefit of VR systems is with flexibility attributes. Different virtual laboratory experiments involving different components (virtual apparatus) can be easily created (Potkonjak et al., 2016). VR labs can be used for experiments that would normally require equipment that is too expensive, complicated, unavailable and unsafe to use in an experiment. The virtual environment can recreate a safe teaching mode that bridges the gaps between traditional laboratories and modern approaches to learning (Chen et al., 2010; Bortnik et al., 2017). ChemCollective and Virtual ChemLab are two examples of virtual laboratories used by chemistry students, funded by the National Science Foundation under the leadership of Dr David Yaron at Carnegie Mellon University (Lerberg, 2008). VR labs also present students' the opportunity to repeat an experiment multiple times, manipulate parameters and settings that could influence the outcome of an experiment (Chen et al., 2010). Similarly, the VR systems enable students to receive immediate feedback to correct a defective understanding of concepts (Tatli \& Ayas 2010; Chen et al., 2010; Bortnik et al., 2017).

Virtual laboratories improve interaction between students and instructors and support discussions between participants in virtual environments. Scheucher et al. (2009) designed a 3D Collaborative Virtual Learning Environment (3D CVLE) for physics education in which students and educators are able to work together in a collaborative way. Virtual laboratories provide instruments for education that are independent from place and time. It is able to carry instruction from closed walls of a classroom to anywhere with a computer and enables applications to become more dynamic with simulations (Tatli \& Ayas, 2010). Furthermore, the VR simulators allow the embedding of performance metrics in the learning software, thus enabling continuous performance feedback (Thomsen et al., 2016). Cheng et al. (2010) designed a collaborative virtual learning environment for children within the autistic spectrum. The 3D empathy system was developed by employing empathy rating scale (ERS) to determine the understanding of empathic behaviours of participants after intervention.

\section{Disadvantages}

Despite the advantages of VR systems in learning environments, they do have their setbacks. Some setbacks of a virtual laboratory system include the requirement to process the expected knowledge into a computer system prior to use (Pearson \& Kudzai, 2015). The VR can create a specific student's attitude such as lack of seriousness, responsibility and carefulness (Potkonjak et al., 2016). At the final stage of training, there is the need to apply real equipment, to be able to acquaint the learners with handson practical experience (Potkonjak et al., 2016).

\section{Application of VR in learning experience}

The extended functionalities that a virtual environment provides in research interest for distance learning has led to the construction of a wide range of applications that implement VR technology in order to sustain the learning process in Educational Virtual Environments (Alexiou et al., 2004). The VR technology is employed in various fields of science, art and education. Due to the rapid development of science and technology, VR technology has been diversified according to the level of interaction and immersion (Ran \& Liu, 2013). The desktop VR system which is an interactive non-immersive system is a low-cost VR system that uses only the personal computers. It uses the computer screen as a window for participant interaction, thereby serving as the virtual environment (Ran \& Liu, 2013). The distributed VR system is another technology that could be maximally applied in the educational field. The distributed VR system is a web-based VR environment which makes use of multiple physical locations in multiple users through network connections (Ran \& Liu, 2013). In this system, the users can share information, work as a team, thereby creating a collaborative workstation and providing the opportunity and necessary technical support for distance learning (Ran \& Liu, 2013). An example of a distributed VR environment for learning is C-VISions. C-VISions is a research project, which focuses on the application of a multi-user 3D environment for educational purposes (Alexiou et al., 2004). Also, Agbonifo et al. (2020) developed a desktop- VR-enabled chemistry laboratory platform for students' adaptive learning - to enable students to learn the titration experiment in a virtual laboratory environment before proceeding to the chemistry wet lab.

\section{Application of VR technology in diverse fields}

The advancement in interactive and immersive technologies had a noticeable impact on various styles of teaching and learning (Abulrub et al., 2011). Likewise, diverse professions have adapted and exploited the VR systems in the advancement of their various fields (Reznek et al., 2002). One area of VR application is in the architectural walkthrough system through the VR visualisation tool (Mazuryk \& Gervautz, 1996). Though it might be impossible to exactly foretell what the future of VR holds, a generalised opinion can be reached on the future of VR systems by examining some ongoing VR research (Guttentag, 2010). In the bit to help students achieve their academic goals, different 
models have been built to achieve learning. Way (2006) presented a model termed Applied Computing Technology Laboratory (ACT Lab) which was built on successful research programs. The ACT Lab is structured for flexibility, efficiency and a dynamic research program that expands the idea of undergraduate students' research activities. The virtual laboratory is a web based platform where students can get access to equipment, guidance or information and that allows collaboration between faculty members and helps create a learning interaction between the teacher and students. Similarly, Belloum et al. (2003) developed a Gridbased Virtual Laboratory Amsterdam (VLAM-G) for learning purposes in applied sciences. The VLAM-G is a grid-based virtual laboratory for remote experimental control and collaborative Grid-based distributed analysis. The need for virtual laboratories arise due to lack of training facilities. Abulrub et al. (2011) demonstrated the use of a 3D interactive VR visualisation system in preparing engineering graduates for practical experience of real industrial environments.

The VR technology has been beneficial in the training of medical students (Abulrub et al., 2011). Rosenthal et al. (2008) reported a high acceptance level of VR while training medical students. The virtual laboratory has helped improve students' skills on laparoscopy and their performance in the operating room. The various tasks performed with the virtual laboratory include: the angled scope task, grasp-andclip task, intracorporeal knotting task and three-dimensional environment task (Rosenthal et al., 2008). The simulationbased training of surgical skills was meant to improve operation performance using VR technology (Thomsen et al., 2016). Proficiency levels have been proven to be among the most effective ways to train in technical skills. One of the numerous benefits of VR application can be found in the health sector or training of students in surgical operations such as training in phlebotomy and treatment of patients for disorders (Wandell, 2010; Carl et al., 2018). An obvious advantage of learning medical procedures by simulated practice is that there is no risk to the lives of patients in the event of a mistake (Wandell, 2010). The EyeSi simulator is a VR simulator used in ophthalmic and cataract surgery. It has an established measure of performance and evidencebased proficiency level (Thomsen et al., 2016). Wandell, (2010) also looked at the effectiveness of VR simulators in phlebotomy. The virtual IV system which is the VR simulation device designed to train students in intravenous fluid line insertion, contains a virtual phlebotomy training. Furthermore, McLay et al. (2010) investigated the beneficial effect of VR technology-aided training for the treatment of post-traumatic stress disorder (PTSD) patients.

VR technology offers a unique opportunity to disseminate exposure therapy (ET) which is a modality for treatment (Carl et al., 2018). McLay et al. (2010) reported the survey of using VR Exposure Therapy (VRET) and the traditional Exposure Therapy (ET), the report showed that all the patients in both groups improved considerably well with an average of $67 \%$ and $50 \%$ respectively. Carl et al. (2018) investigated the acceptability of the VRET and traditional ET technology in medicine, and showed that $76 \%$ of respondents opt for VRET over traditional ET for treatment, while $81-89 \%$ of college students preferred VRET over traditional ET. Carl et al. (2018) also reported the success of VRET in treating specific phobias, such as: fear of flying (aerophobia), fear of heights (acrophobia) and fear of animals (zoophobia).

The VR machinery and software consist of J\&J Engineering biofeedback systems which have the ability to substitute the portable biofeedback units (StressErasers). The VRET function in such a way that the client computer provided 3D images through a head-mounted display (HMD) and a joystick controller to allow the client to move and interact with the stimulated world (McLay et al., 2010). Guttentag (2010), investigated the application of VR technology to tourism and its implication. The VR technology was applied in the aviation industry for effective transfer of safety knowledge to passengers on board an airline. This has been successfully disseminated through mobile VR on personal electronic devices (PEDs) such as smartphones and tablets of most passengers on-board (Chittaro et al., 2018). The interactive content of the VR technology was sent alongside with electronic tickets or boarding passes to passengers through their smartphones. Chittaro et al. (2018) explored the possible effectiveness of VR approach in making interactive safety briefings. The mobile VR application referred to as app was developed using Unity 4.5 game engine and C\# programming language which shows a full 3D aircraft cabin environment (Chittaro et al., 2018).

Virtual manufacturing is defined as a computer system which is capable of generating information about the structure, status, and behaviour of a manufacturing system as can be observed in a real manufacturing environment (Mujber et al., 2004). VR in manufacturing is applied in design, prototyping, machining, assembly, inspection, planning, training and simulation (Mujber et al., 2004). The VR provides designers with a virtual environment at the concept design stage in the design of a product. The virtual prototyping is used in designing before the physical prototype is used to prove the design, alternatives, to do engineering analysis, manufacturing planning, support management decisions, and to get feedback on a new product from prospective customers (Mujber et al., 2004). Hadi et al., (2011) demonstrate the use of a 3D interactive VR visualisation system in preparing engineering graduates for practical experience of real industrial environments. Balogun et al. (2010) explored virtual tourism by designing a 3D Geo-spatial VR system for tourist centres and historical heritage to help manage and promote tourism thereby increasing the Gross Domestic Product (GDP) of the country.

Dixon (2006) surveys the history of various VR technologies used in the field of theatrical and performance arts. Some of the VR technologies are Placeholder, Osmose, Virtual Bodies, VR scenography in real time, ieVR's Mechanical and Blast Theory's Desert Rain. Placeholder premiered in 1993 is the much-celebrated project by Brenda Laurel and Rachel Strickland that opened up potential for virtual flight. Osmose (1994-1995), created by Char Davies, reported the VR system as a revolutionary fully embodied immersion technology which uses sophisticated data-suits to sense living immersive experience. Virtual Bodies: Dancing with the aid of Virtual Dervish (1994) was developed by Yacov Sharir and Diane Gromala, it is a visual body programmed in a continuous motion and undulates as if breathing like a living body. The Institute for the Exploration of VR used 
VR tools to recreate and remediate theatre history. Also, VR technology is used as a prime scenographic medium to achieve a sense of immersion. Machinal (1999) used the staging configuration of ieVR's first production to pre-record moving 3D imagery of landscapes, objects and hyper-realistic machinery. Finally, Blast Theory's Desert Rain (1999) is the closest to VR performance event due to its aesthetic form which render it the most innovative and futuristic utilisation of performance VR.

\section{Prospect of VR}

The VR technology is invariably advancing with an increasing development of more systems to help improve human experiences. Studies are ongoing by researchers on six degrees of freedom (6DoF) positions and orientationaware computer interfaces to support access to embedded information attached to the physical world all around us (Welch, 2009). The benefit will be employed in laboratories, the hallway, parks, city sidewalks and individuals will in the future see, hear, and interact with information that exists as an essential part of their immediate physical environment (Welch, 2009). With this advancement, the medical field will also be a benefactor of its numerous benefits, as doctors could be able to remotely treat patients through an image like a surrogate (medical avatar). The VR technology holds an advantage to therapists to be able to provide a wide range of controlled stimuli and monitor responses to treatment by patients. Workers and technicians can be directed on assembling and maintenance through VR technology. The blind can be given gaze-directed aural sight, and deaf people visual hearing through VR technology (Riva, 2003; Welch, 2009). Information and associated databases will be structured by physical location and time, enabling users to both store and retrieve past, present, and future information in the framework of physical locality and direction of gaze (Welch, 2009). In the education sector, visual education can include synchronous face-to-face interaction, group interaction, voice communication, examination of 3D models, projection of visual information in a PowerPoint window (Messinger et al., 2009). Also according to Dixon (2006), the potential of VR technology can be used for dance engagement in theatre and arts. In the teaching of history in school VR tools can help provide avenues for reconceptualisation of the past with some energy and excitement in the field (Allison, 2008).

\section{Conclusion}

VR technology will continue to have a beneficial role in various fields of study and training. In general, the review of current applications shows that VR can be considered a useful tool for diagnosis, therapy, education and training however, several barriers still remain. The PC-based systems, while being inexpensive and easy-to-use, still suffer from a lack of flexibility (Riva, 2003). It is obvious that VR can be used as an alternative in learning activities and proffering solutions to some challenges associated with inadequacy of equipment or facilities in the learning environment. The medical field has been able to apply VR in various aspects such as in training of medical students and treatment of diseases. The aviation industry has used the VR systems to aid the effective dissemination of information to passengers on-board in air transit. It also gives confidence to students to carry out the practical aspect of their studies without any physical damage and harm.

\section{References}

Abulrub, A.-H. G., Attridge, A. N., \& Williams, A. M. (2011). Virtual reality in engineering education: The future of creative learning. IEEE Global Engineering Education Conference (EDUCON). Learning Environments and Ecosystems in Engineering Education, 751 - 757.

Agbonifo O.C, Sarumi O.A, \& Akinola Y.M (2020). A virtual reality enabled chemistry laboratory platform for students' adaptive learning. Research in Learning Technology. Preprint

Alexiou, A., Bouras, C., \& Giannaka, E. (2004). Virtual laboratories in education. In IFIP World Computer Congress, TC 3, (19-28). Springer, Boston, MA.

Allison, J. (2008). History educators and the challenge of immersive pasts: A critical review of virtual reality 'tools' and history pedagogy. Learning, Media and Technology, 33(4), 343-352.

Baarspul, M. (1990). A review of flight simulation techniques. Progress in Aerospace Sciences, 27(1), 1-120.

Babateen, H. M. (2011). The role of virtual laboratories in science education. In 5th International Conference on Distance Learning and Education IPCSIT, 12, 100-104.

Balogun, V. F., Thompson, A. F., \& Sarumi, O. A. (2010). A $3 \mathrm{D}$ geo-spatial virtual reality system for virtual tourism. The Pacific Journal of Science and Technology, 11(2), 601-609.

Belloum, A. S., Groep, D. L., Hendrikse, Z. W., Hertzberger, B. L., Korkhov, V., Laat, C. T., \& Vasunin, D. (2003). VLAM-G: A grid-based virtual laboratory. Future Generation Computer Systems, 19(2), 209 - 217.

Boas, Y. A. G. V. (2013). Overview of virtual reality technologies. In Interactive Multimedia Conference, 1 - 6.

Bortnik, B., Stozhko, N., Pervukhina, I., Tchernysheva, A., \& Belysheva, G. (2017). Effect of virtual analytical chemistry laboratory on enhancing student research skills and practices. Research in Learning Technology, 25, 1-20.

Carl, E., Stein, A. T., Levihn-Coon, A., Pogue, J. R., Rothbaum, B., Emmelkamp, P., \& Powers, M. B. (2019). Virtual reality exposure therapy for anxiety and related disorders: A metaanalysis of randomized controlled trials. Journal of anxiety disorders, 61, 27-36.

Chen, X., Song, G., \& Zhang, Y. (2010). Virtual and Remote Laboratory Development: A Review. In Earth and Space 2010: Engineering, Science, Construction, and Operations in Challenging Environments, 3843 - 3852. 
Cheng, Y., Chiang, H. C., Ye, J., \& Cheng, L. H. (2010). Enhancing empathy instruction using a collaborative virtual learning environment for children with autistic spectrum conditions. Computers \& Education, 55(4), 14491458.

Chittaro, L., Corbett, C. L., McLean, G. A., \& Zangrando, N. (2018). Safety knowledge transfer through mobile virtual reality: A study of aviation life preserver donning. Safety science, $102,159-168$.

Chow, A., Andrews, S., and Trueman, R. (2007). A 'Second Life': can this online, virtual reality world be used to increase the overall quality of learning and instruction in graduate distance learning programs. In Proceedings of the Association for Educational Communications and Technology International Convention. (Vol 2, 75-83). Bloomington, IN: Association for Educational Communications and Technology.

Desai, K., Belmonte, U. H. H., Jin, R., Prabhakaran, B., Diehl, P., Ramirez, V. A., \& Gans, M. (2017, April). Experiences with multi-modal collaborative virtual laboratory (mmcvl). In 2017 IEEE Third International Conference on Multimedia Big Data (BigMM) (376-383). IEEE.

Dixon, S. (2006). A history of virtual reality in performance. International Journal of Performance Arts \& Digital Media, $2(1), 23-54$.

Drummond, K., Houston, T., \& Irvine, T. (2014). The rise and fall and rise of virtual reality. Vox Media.

Egger, J., Gall, M., Wallner, J., Boechat, P., Hann, A., Li, X., Chen, X \& Schmalstieg, D. (2017). HTC Vive MeVisLab integration via OpenVR for medical applications. PloS one, 12(3), 1-14.

Ellis, S. R. (1994). What are virtual environments?. IEEE Computer Graphics and Applications, 14(1), 17-22.

Guttentag, D. A. (2010). Virtual reality: Applications and implications for tourism. Tourism Management, 31(5), 637651.

Holden, M. K. (2005). Virtual environments for motor rehabilitation. Cyberpsychology \& behavior, 8(3), 187-211.

Hui-Zhen, R., \& Zong-Fa, L. (2013). Application and prospect of the virtual reality technology in college ideological education. Fourth International Conference on Intelligent Systems Design and Engineering Applications, 125-128. IEEE.

Kumar, D. (2014). Virtual Reality in the Real World. IJRIT International Journal of Research in Information Technology, 2(4), 309- 313.

Lambropoulos, N. (2007). User-centered design of online learning communities. In User-centered design of online learning communities, 1-28. IGI Global

Lerberg, B. (2008). Virtual labs in a chemistry classroom. OpenStax-CNX module: 1-5.

Markwalter, B. (2014). Entertainment and immersive content:
What's in store for your viewing pleasure. IEEE Consumer Electronics Magazine, 4(1), 83-86.

Martirosov, S., \& Kopecek, P. (2017). Virtual reality and its influence on training and education-literature review. Annals of DAAAM \& Proceedings, 28.

Mazuryk, T., \& Gervautz, M. (1996). Virtual reality-history, applications, technology and future, 1-72.

McLay, R. N., McBrien, C., Wiederhold, M. D., \& Wiederhold, B. K. (2010). Exposure therapy with and without virtual reality to treat PTSD while in the combat theater: A parallel case series. Cyberpsychology, behavior, and social networking, 13(1), 37-42.

Messinger, P. R., Stroulia, E., Lyons, K., Bone, M., Niu, R. H., Smirnov, K., \& Perelgut, S. (2009). Virtual worlds-past, present, and future: New directions in social computing. Decision support systems, 47(3), 204-228.

Mun, S., Whang, M., Park, S., Lee, D. W., \& Kim, H. I. (2018), Overview of VR Media Technology and Methods to Reduce Cybersickness. Journal of Broadcast Engineering, 23(6), 800812.

Mujber, T. S., Szecsi, T., \& Hashmi, M. S. (2004). Virtual reality applications in manufacturing process simulation. Journal of materials processing technology, 155, 1834-1838.

Mykhailovska, O. V., Chervynskyi, A. I., \& Fokin, D. P. (2019). The prospects of Virtual Reality (VR) technology in educational process and business. Науковий вісник Полісся, (1), 139-147.

Potkonjak, V., Gardner, M., Callaghan, V., Mattila, P., Guetl, C., Petrović, V. M., \& Jovanović, K. (2016). Virtual laboratories for education in science, technology, and engineering: $A$ review. Computers \& Education, 95, 309-327.

Reznek, M., Harter, P., \& Krummel, T. (2002). Virtual reality and simulation: training the future emergency physician. Academic Emergency Medicine, 9(1), 78-87.

Riva, G. (2003). Applications of virtual environments in medicine. Methods of information in medicine, 42(05), 524534.

Rizzo, A. A., Graap, K., Perlman, K., McLAY, R. N., Rothbaum, B. O., Reger, G., Parsons,T., Difede, J \& Pair, J. (2008). Virtual Iraq: initial results from a VR exposure therapy application for combat-related PTSD. Studies in health technology and informatics, 132, 420.

Rosenthal, R., Gantert, W. A., Hamel, C., Metzger, J., Kocher, T., Vogelbach, P., \& Hahnloser, D. (2008). The future of patient safety: Surgical trainees accept virtual reality as a new training tool. Patient safety in surgery, 2(1), 16.

Rundle, A. G., Bader, M. D., Richards, C. A., Neckerman, K. M., \& Teitler, J. O. (2011). Using Google Street View to audit neighborhood environments. American journal of preventive medicine, 40(1), 94-100. 
Scheucher, B., Bayley, P., Gütl, C., \& Harward, J. (2009). Collaborative virtual $3 d$ environment for internetaccessible physics experiments. International Journal of Online Engineering, 5(REV 2009), 65-71.

Schmid, C. (2007). Grid technologies for virtual laboratories in engineering education. International Journal of Online Engineering (iOE), 4(1).

Shudayfat, E., Moldoveanu, F., \& Moldoveanu, A. (2012). A $3 D$ virtual learning environment for teaching chemistry in high school. In Annals of DAAAM for 2012 \& Proceedings of the 23rd International DAAAM Symposium (Vol. 23, No. 1, 2304-1382).

Standen, P. J., \& Brown, D. J. (2006). Virtual reality and its role in removing the barriers that turn cognitive impairments into intellectual disability. Virtual Reality, 10(3-4), 241-252.

Steuer, J. (1992 ). Defining Virtual Reality: Dimensions Determining Telepresence. Journal of Communication, Autumn 42(4), 73-93.

Sveistrup, Heidi. "Motor rehabilitation using virtual reality." Journal of neuroengineering and rehabilitation 1.1 (2004): 10.

Tatli, Z., \& Ayas, A. (2010). Virtual laboratory applications in chemistry education. Procedia-Social and behavioral sciences, 9, 938-942.
Thomsen, A. S. S., Bach-Holm, D., Kjærbo, H., Højgaard-Olsen, K., Subhi, Y., Saleh, G. M., \& Konge, L. (2017). Operating room performance improves after proficiency-based virtual reality cataract surgery training. Ophthalmology, 124(4), 524-531.

Way, T. P. (2006). A virtual laboratory model for encouraging undergraduate research. ACM SIGCSE Bulletin, 38(1), 203207.

Welch, G. F. (2009). History: The use of the kalman filter for human motion tracking in virtual reality. Presence: Teleoperators and Virtual Environments, 18(1), 72-91.

Youngblut, C. (1998). Educational uses of virtual reality technology(No. IDA-D-2128). Institute for defense analysis, Alexandria VA.

Zachara, M., \& Zagal, J. P. (2009). Challenges for success in stereo gaming: A Virtual Boy case study. In Proceedings of the international conference on Advances in Computer Enterntainment Technology (99-106).

Zurweni, Wibawa, B., \& Erwin, T. N. (2017). Development of collaborative-creative learning model using virtual laboratory media for instrumental analytical chemistry lectures. In American Institute of Physics Conference Proceedings Nol. 1868, No. 1, 030010). AIP Publishing LLC.

Copyright: @ 2020 Yewande M. Akinola, Oluwatoyin C. Agbonifo, and Oluwafemi A. Sarumi. This is an open-access article distributed under the terms of the Creative Commons Attribution License (CC BY). The use, distribution or reproduction in other forums is permitted, provided the original author(s) and the copyright owner(s) are credited and that the original publication in this journal is cited, in accordance with accepted academic practice. No use, distribution or reproduction is permitted which does not comply with these terms. 